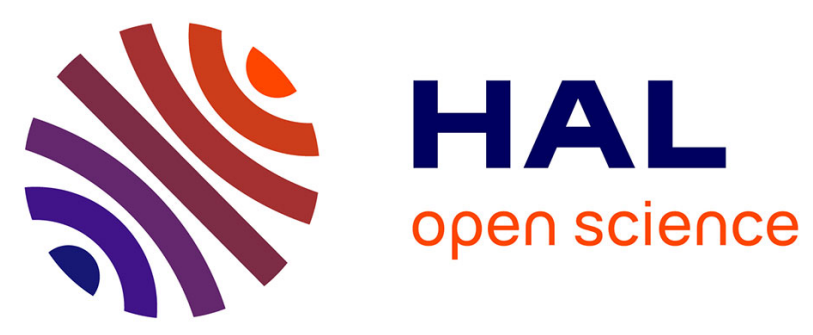

\title{
Preface to the special issue on Graph Searching: Theory and Applications
}

Spyros Angelopoulos, Nancy Clarke, Fedor Fomin, Archontia Giannopoulou, Roman Rabinovich

\section{- To cite this version:}

Spyros Angelopoulos, Nancy Clarke, Fedor Fomin, Archontia Giannopoulou, Roman Rabinovich. Preface to the special issue on Graph Searching: Theory and Applications. Theoretical Computer Science, 858, Elsevier, pp.145-146, 2021, 10.1016/j.tcs.2021.01.036 . hal-03439955

\section{HAL Id: hal-03439955 https://hal.science/hal-03439955}

Submitted on 22 Nov 2021

HAL is a multi-disciplinary open access archive for the deposit and dissemination of scientific research documents, whether they are published or not. The documents may come from teaching and research institutions in France or abroad, or from public or private research centers.
L'archive ouverte pluridisciplinaire HAL, est destinée au dépôt et à la diffusion de documents scientifiques de niveau recherche, publiés ou non, émanant des établissements d'enseignement et de recherche français ou étrangers, des laboratoires publics ou privés. 


\section{Preface to the special issue on Graph Searching: Theory and Applications}

The idea of this special issue of Theoretical Computer Science originates from the 9th International Workshop on GRAph Searching: Theory and Applications (GRASTA 2018) that took place in Berlin, Germany between the 24th and 27th of September, 2018. The issue contains results that were presented during GRASTA 2018 as well as external contributions on Graph Searching. GRASTA 2018 took place in Berlin, Germany between the $24^{\text {th }}$ and $27^{\text {th }}$ of September, 2018. The workshop was established in 2006 and since then it has traveled around the world, being held in Anogia, Crete, Greece (2006 and 2017), Praia de Redonda, Ceara, Brazil (2008), Valtice chateau, Valtice, Czech Republic (2009), Dagstuhl, Germany (2011), Banff, Canada (2012), Cargèse, Corsica, France (2014), Montréal, Canada (2015) (joint with 5th workshop on Moving And Computing (MAC)) and Berlin, Germany (2018). It has become a regular meeting where results within the broad scope of Graph Searching are communicated and collaborations are promoted between a wide range of researchers from all over the world.

Graph Searching can be characterised as a game played by two opposing teams inside a network, modelled by a graph. The first team consists of mobile agents (called searchers or pursuers or cops) and the second team consists of escaping agents (called evaders or fugitives or robbers) residing and moving within the graph. Both the mobile and the escaping agents can be assigned attributes picking from a wide pool of options, including their respective relative speeds, sensor capabilities, visibility, etc. The graph searching games may then take vastly different forms. The pursuers win if they capture the evaders. Otherwise, the evaders win. The notion of capture itself admits several interpretations as well.

Many game variants have been studied in the literature and are either application driven or are inspired by foundational issues in Computer Science, Discrete Mathematics, and Artificial Intelligence. Moreover, they are related to diverse topics such as Robot Motion Planning, Database Theory, Logic, Distributed Computing, Models of Computation, and Network Security.

This Special Issue received 11 submissions in total. All submissions were reviewed according to the highest standards of Theoretical Computer Science, and among them 7 submissions were finally accepted for publication. The selected papers cover a wide range of topics such as: Capture Time Complexities of Graph Searching Games, Directed Graph Theory, Discrete Optimization, Game Theory, Graph Admissibility, Graph Decomposition Theorems, Online Algorithms and Scheduling.

A more detailed description of the accepted papers, in order of final acceptance date, is as follows.

- In the paper A search game on a hypergraph with booby traps, Thomas Lidbetter and Kyle Y. Lin consider the graph searching game that follows. A set of $n$ boxes, located on the vertices of a hypergraph $G$, contain known but different rewards. A Searcher opens all the boxes in some hyperedge of $G$ with the objective of collecting the maximum possible total reward. Some of the boxes, however, are booby trapped. If the Searcher opens a booby trapped box, the search ends and she loses all her collected rewards. The authors assume the number $k$ of booby traps is known, and model the problem as a zero-sum game between the maximizing Searcher and a minimizing Hider, where the Hider chooses $k$ boxes to booby trap and the Searcher opens all the boxes in some hyperedge. The payoff is the total reward collected by the Searcher. The authors solve the game when $G$ is a 1-uniform hypergraph (the hyperedges are all singletons). When $G$ is the complete hypergraph (containing all possible hyperedges), the game is solved in the cases where each box has the same reward, $k=1$, and finally $n=4$ and $k=2$. 
- In the paper Edge degeneracy: Algorithmic and structural results, the authors Stratis Limnios, Christophe Paul, Joanny Perret, and Dimitrios M. Thilikos consider a cops and robber game where the cops are blocking edges of a graph $G$, while the robber occupies its vertices. At each round of the game, the cops choose some set of edges to block and right after the robber is obliged to move to another vertex traversing at most $s$ unblocked edges ( $s$ can be seen as the speed of the robber). Both parts have complete knowledge of the opponent's moves and the cops win when they occupy all edges incident to the robber's position. The authors introduce the capture cost on $G$ against a robber of speed $s$. This defines a hierarchy of invariants, namely $\delta_{e}^{1}, \delta_{e}^{2}, \ldots, \delta_{e}^{\infty}$, where $\delta_{e}^{\infty}$ is an edge-analogue of the admissibility graph invariant. They prove that the problem asking whether $\delta_{e}^{s}(G) \leq k$, is polynomially solvable when $s \in\{1,2, \infty\}$ while, otherwise, it is NP-complete. Their main result is a structural theorem for graphs of bounded edge-admissibility.

- Sebastian Brandt, Klaus-Tycho Foerster, Jonathan Maurer, and Roger Wattenhofer in their paper Online graph exploration on a restricted graph class: Optimal solutions for tadpole graphs study the problem of online graph exploration on undirected graphs, where a searcher has to visit every vertex and return to the origin. Once a new vertex is visited, the searcher learns of all neighboring vertices and the connecting edge weights. The goal of such an exploration is to minimize its total cost, where each edge traversal incurs a cost of the corresponding edge weight. They investigate the problem on tadpole graphs (also known as dragons, kites), which consist of a cycle with an attached path.

- The paper A tight lower bound for the capture time of the Cops and Robbers game by Sebastian Brandt, Yuval Emek, Jara Uitto, and Roger Wattenhofer proves that the upper bound $\mathcal{O}\left(n^{k+1}\right)$ on the capture time in $k$-cop-win graphs is at most asymptotically tight for any constant $k \geq 2$, answering in the negative an open question of Bonato and Nowakowski who asked whether this upper bound could be improved. Their result yields a surprising gap in the capture time complexities between the 1-cop and the 2-cop cases since in 1-cop-win graphs, the cop can capture the robber in $O(n)$ time.

- Masood Masjoody and Ladislav Stacho in their paper Cops and robbers on graphs with a set of forbidden induced subgraphs characterize all sets $\mathcal{H}$ of graphs with bounded diameter, such that $\mathcal{H}$ free graphs are cop-bounded. This gives a characterization of cop-bounded classes of graphs defined by a finite set of connected graphs as forbidden induced subgraphs. Furthermore, they extend the characterization to the case of cop-bounded classes of graphs defined by a set $\mathcal{H}$ of forbidden graphs such that the components of members of $\mathcal{H}$ have bounded diameter.

- In the paper Simple strategies versus optimal schedules in multi-agent patrolling, the authors Akitoshi Kawamura and Makoto Soejima consider the cops and robbers game where mobile agents are assigned different maximum speeds and their goal is to patrol a fence together so as to minimize the longest time interval during which a point on the fence is left unvisited. The authors consider the case where the fence is an interval and a circle and construct schedules that are 4/3 times (for Interval Patrolling) and 21/20 times (for Circle Patrolling) as good, respectively, as the simple strategies proposed in the literature. They also propose a new variant, in which the goal is to patrol a single point under the constraint that each agent can only visit the point some predefined time after its previous visit and obtain some similar ratio bounds and NP-hardness results related to this problem.

- Sebastián González Hermosillo de la Maza, Seyyed Aliasghar Hosseini, Fiachra Knox, Bojan Mohar, and Bruce Reed in their paper Cops and robbers on oriented toroidal grids consider the straight-ahead orientations of 4-regular quadrangulations of the torus and the Klein bottle and prove that their cop number is bounded by a constant. They also show that the cop number of every $k$-regularly oriented toroidal grid is at most 13 .

We are hopeful that the results selected for presentation in this Special Issue will contribute to further advances in the field of Graph Searching.

Guest Editors 
Spyros Angelopoulos CNRS and Sorbonne University, Paris, France

Nancy Clarke Acadia University, Wolfville, Nova Scotia, Canada

Fedor Fomin

The University of Bergen, Bergen Norway Archontia C. Giannopoulor National and Kapodistrian University of Athens, Athens, Greece

Roman Rabinovich Technische Universität Berlin, Berlin, Germany

\footnotetext{
${ }^{1}$ Corresponding Editor, E-mail address: arcgian@di.uoa.gr
} 